\title{
The Hodgkin-Huxley neuron model on the fast phase plane
}

\author{
William Aristizabal Botero ${ }^{1}$, Alvaro H. Salas ${ }^{1 \star}$ and Silvia Janeth Gonzalez Colorado ${ }^{2}$ \\ ${ }^{1}$ Universidad de Caldas, Universidad Nacional de Colombia, FIZMAKO Research Group, Colombia. \\ ${ }^{2}$ 2Universidad Nacional Abierta y a Distancia, UNAD, Faculty of Psychology, Pereira Colombia.
}

Accepted 14 May, 2012

\begin{abstract}
In this paper, we showed a simplified version of the Hodgkin-Huxley neuron model, the so called fast plane-phase case. We first describe the circuit model of axon membrane with four parallel branches described by Hodgkin and Huxley and then we apply the Kirchhoff's laws to obtain a system ordinary differential equations. We obtain numerical solutions to it with the aid of Mathematica. We also study this simplified model from the point of view of dynamical systems. With the aid of Maple we graph the phase portrait corresponding to the dynamical system associated to Hodgkin-Huxley model. At the end we interpret the results.
\end{abstract}

Key words: Neuron model, Hodgkin-Huxley neuron model, cell membrane, ionic channel dynamics, fast phase plane, nullclines, mathematical.

\section{INTRODUCTION}

In the study of Hodking et al. (1952A), Hodking and Huxley (1952 B,C,D,E) (H.H for short) were the first quantitative model about generation and propagation of action potential through excitable membrane along giant squid axon and their ideas have been extended and applied to a variety of excitable cells (Ebihara and Johnson, 1980; Luo and Rudy, 1994). The H.H model is the cornerstone of electrophysiology and has been used by a wide group of researchers. A simplified model postulated simultaneously by Fitzhugh (1969) and Nagumo et al. (1962) originated a new field in applied Mathematics. This model showed that the essentials of the excitable process could be understood in terms of a simpler model upon which Mathematical analysis could make some progress. There are other models in the literature that deals with action potential like the ConnorStevens model that provides an alternative description of action potential generation in isolated neural somata of marine gastropods (Connor and Stevens, 1971).

\section{ELECTRICAL CIRCUIT AND THE HODGKIN-HUXLEY MODEL}

Figure 1 represents an equivalent circuit model of axon membrane with four parallel branches described by Hodgkin and Huxley (1952), which shows the $\mathrm{Na}^{+}, \mathrm{K}^{+}$ ionic channels and the leakage ionic channels $L$, which may include $\mathrm{Cl}^{-}$and other ion flows. $C_{m}$ represents the thin dielectric properties of the membrane (Hille, 2001). For simplicity, all channels of the same type are lumped in one single channel each and the lipid layer is presented as a single capacitor. Since $\mathrm{K}^{+}$has a higher concentration inside than outside the cell, the positive $\mathrm{K}^{+}$ ions will flow outward of the membrane. The reverse situation prevails for positive $\mathrm{Na}^{+}$ions. Namely, ions have a tendency to move down their concentration gradients. Such a tendency is denoted in the circuit by the batteries with voltages $E_{N a}, E_{K}$ and $E_{L}$. These voltages depend on the inside-outside concentration difference in each ion 


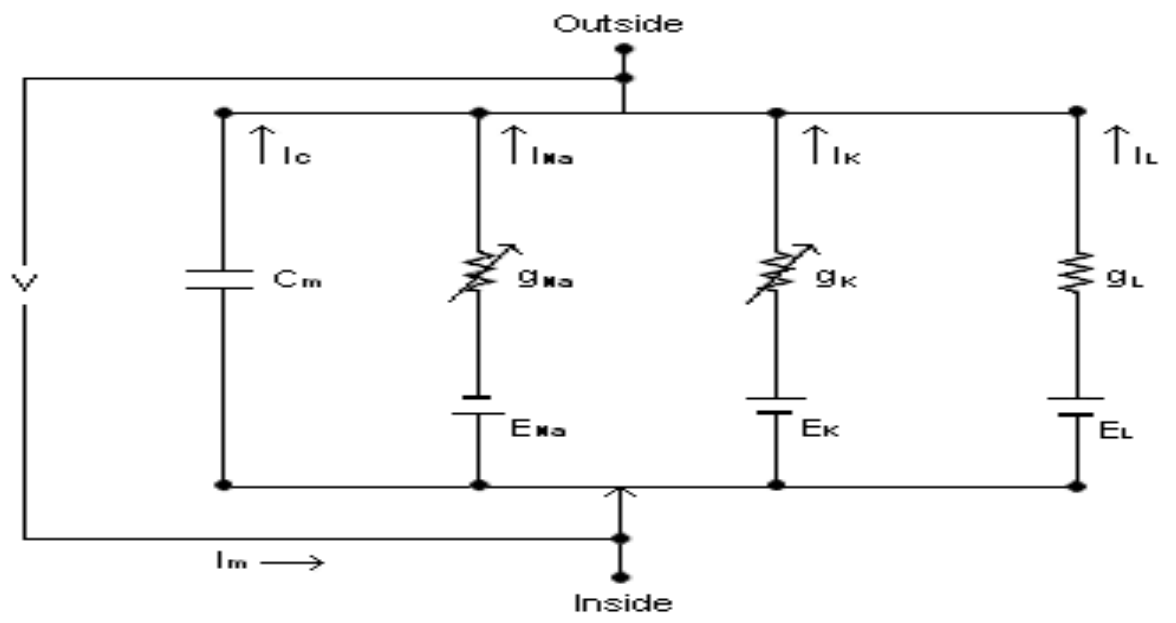

Figure 1. Equivalent circuit of axon membrane.

(Hille, 2001). It was noticed in the circuit that the polarities of the $\mathrm{Na}^{+}$and $\mathrm{K}^{+}$batteries are reversed but $\mathrm{K}^{+}$ and $\mathrm{L}$ batteries have the same polarities.

If $g_{\mathrm{Na}}$ and $g_{K}$ are the voltage and time dependent conductivities of $\mathrm{Na}^{+}, \mathrm{K}^{+}$channels, and $g_{L}$ represents the $L$ ions leak conductance and this last one is considered constant, then, it is possible to write the following set of equations based on the aforementioned circuit analysis in Figure 1. Applying the Kirchhoff's laws to the aforementioned circuit, the following Equations can be obtained:

$$
\begin{aligned}
& I_{m}=C_{m} \frac{d v}{d t}+I_{K}+I_{N a}+I_{L} . \\
& I_{K}=g_{K}\left(v-E_{K}\right) \\
& I_{N a}=g_{N a}\left(v-E_{N a}\right) . \\
& I_{L}=g_{L}\left(v-E_{L}\right) .
\end{aligned}
$$

In these Equations, $v$ is the action potential, $I_{m}$ is the current injected into the axon and $E_{N a}, E_{K}$ and $E_{L}$ are the Nernst's equilibrium potentials for each of the ions considered. Thus, Equation (1) may be written in the form of:

$$
I_{m}=C_{m} \frac{d v}{d t}+g_{K}\left(v-E_{K}\right)+g_{N a}\left(v-E_{N a}\right)+g_{L}\left(v-E_{L}\right)
$$

In Equation (5), $g_{L}$ is considered voltage-independent and thus, this parameter is a constant. However, it was demonstrated by Hogking and Huxley (1952) in their study that $g_{N a}$ and $g_{K}$ depends on other parameters related to ionic channel dynamics and they fitted these relations with their experimental information. The complete set of equations that were obtained by $\mathrm{H} . \mathrm{H}$ is fully presented in some works by several authors Keener et al. (1998); Waxman et al. (1995); Edelstein et al. (1988); Schierwagen (1991); Scott (2002) and Doi et al. (2010).

Fitzhugh's approaches to H.H model takes into account the fact that some variables in this model have fast kinetics and other variables have slower kinetics. This is based on the fact that sodium $m$ gates are activated quickly and as such, the membrane potential $v$ changes quickly too, at least during certain time intervals. On the other hand, potassium $n$ gates and sodium $h$ gates, which describe the falling phase of $g_{N a}$ presents slower processes (that is, $\mathrm{Na}^{+}$channels are inactivated slowly, and $\mathrm{K}^{+}$channels activated slowly). Thus, during the initial stages of the action potential, $n$ and $h$ remain essentially constant while $m$ and $v$ varies (Keener et al., 1998; Edelstein et al., 1988; Scott, 2002). Due to these statements, during start of action potential, it is possible to consider that $n$ and $h$ processes are constant and thus, the four differential equations of the H.H model are reduced to two equations which permits the making of the analysis in a $v-m$ two-dimensional phase-space, named the fast phase-plane or reduced phase-plane (Keener et al., 1998; Edelstein et al., 1988). The HH model is reduced to:

$$
\left\{\begin{array}{l}
\frac{d v}{d t}=-\frac{1}{C_{m}}\left[\bar{g}_{k} n_{0}^{4}\left(v-E_{K}\right)+\bar{g}_{N a} m^{3}(t) h_{0}\left(v-E_{N a}\right)+g_{L}\left(v-E_{L}\right)\right] . \\
\frac{d m}{d t}=\alpha_{m}(1-m(t))-\beta_{m} m(t),
\end{array}\right.
$$


Where $\bar{g}_{N a}$ and $\bar{g}_{K}$ are constants that represent the sodium and potassium maximum conductance, respectively. The variables $m, n$ and $h$ take a (dimensionless) value between zero and unity, and are called the gate variables (Doi et al., 2010). The parameters $n_{0}$ and $h_{0}$ are constants that represent the resting states of the slow variables $n$ and $h$. In addition, the quantities $\alpha_{m}$ and $\beta_{m}$ are assumed to be voltagedependent and represent the dynamic opening and closing of gates that obey a simple process described as follows:

$\alpha_{m}=\alpha_{m}(v)=\frac{\frac{1}{10}(v+40)}{1-\exp \left(-\frac{v+40}{10}\right)}$ and $\beta_{m}=\beta_{m}(v)=4 \exp \left(-\frac{v+65}{18}\right)$

These equations allowed us to study the solutions of the $\mathrm{HH}$ simplified model and obtain, from the point of view of dynamical systems, the action potential dynamic results in the first stage, called depolarization. This simplified model can be most easily studied in the two-dimensional system, $(v$ and $m)$ phase-plane which provides a useful way to study the start of the sodium fast channels dynamic in the action potential generation.

\section{GENERAL FACTS ABOUT AUTONOMOUS SYSTEMS}

Equation (6) has the form:

$$
\left\{\begin{array}{l}
\frac{d v}{d t}=F(v, m) \\
\frac{d m}{d t}=G(v, m)
\end{array}\right.
$$

Where;

$$
F(v, m)=\frac{1}{C_{m}}\left(\bar{g}_{N a} h_{0} m^{3}\left(v-E_{N a}\right)+\bar{g}_{K} n_{0}^{4}\left(v-E_{K}\right)+g_{L}\left(v-E_{L}\right)\right)
$$

and

$$
G(v, m)=\frac{\frac{1}{10}(v+40)}{1-\exp \left(-\frac{v+40}{10}\right)}(1-m)-4 m \exp \left(-\frac{v+65}{18}\right)
$$

The next two curves are called nullclines:

$$
F(v, m)=0 \quad \text { and } \quad G(v, m)=0
$$

Any point at which these curves meet is called an equilibrium point. In order to find the equilibrium points, we must solve following system:

$\left\{\begin{array}{l}F(v, m)=0 \\ G(v, m)=0\end{array}\right.$

Let $\left(v_{e}, m_{e}\right)$ be an equilibrium point of system (12). The jacobian matrix at this point is given by:

$$
J_{e}=\left(\begin{array}{ll}
\frac{\partial F}{\partial v}\left(v_{e}, m_{e}\right) & \frac{\partial F}{\partial m}\left(v_{e}, m_{e}\right) \\
\frac{\partial G}{\partial v}\left(v_{e}, m_{e}\right) & \frac{\partial G}{\partial m}\left(v_{e}, m_{e}\right)
\end{array}\right)
$$

Let:

$\operatorname{Det}=\operatorname{Det}\left(J_{e}\right)$ and $\operatorname{Tr}=\operatorname{Trace}\left(J_{e}\right)=\frac{\partial F}{\partial v}\left(v_{e}, m_{e}\right)+\frac{\partial G}{\partial m}\left(v_{e}, m_{e}\right)$

and

$\delta=\operatorname{Tr}^{2}-4$ Det

Figure 2 shows the parabola $\delta=0$, that is Det $=\frac{1}{4} \operatorname{Tr}^{2}$ and the distinct possibilities for the equilibrium point $\left(v_{e}, m_{e}\right)$ in the so called trace-determinant plane.

\section{SOLUTIONS TO THE PROPOSED MODEL}

Equation (8) is highly non-linear. It does not admit exact solutions. However, we may solve it numerically with the aid of Mathematical, version 7. The code is given in the Appendix. We are going to solve it by using the following parameter values:

$$
\begin{aligned}
& C_{m}=1 \frac{\mu F}{\mathrm{~cm}^{2}}, g_{L}=0.3 \frac{\mathrm{mS}}{\mathrm{cm}^{2}}, E_{L}=-54.4 \mathrm{mV}, E_{K}=-77 \mathrm{mV}, \\
& \bar{g}_{K}=36 \frac{\mathrm{ms}}{\mathrm{cm}^{2}}, \bar{g}_{N a}=120 \frac{\mathrm{mS}}{\mathrm{cm}^{2}}, E_{N a}=50 \mathrm{mV}, n_{0}=0.32, h_{0}=0.45
\end{aligned}
$$

The initial conditions are:

$$
v(0)=-66 \text { and } m(0)=0.01
$$

From Equation (12) and taking into account (9) and (10) we obtain the following equations for the nullclines (we solve the first equation $F(v, m)=0$ for $v$ and the second one $G(v, m)=0$ for $m)$ : 

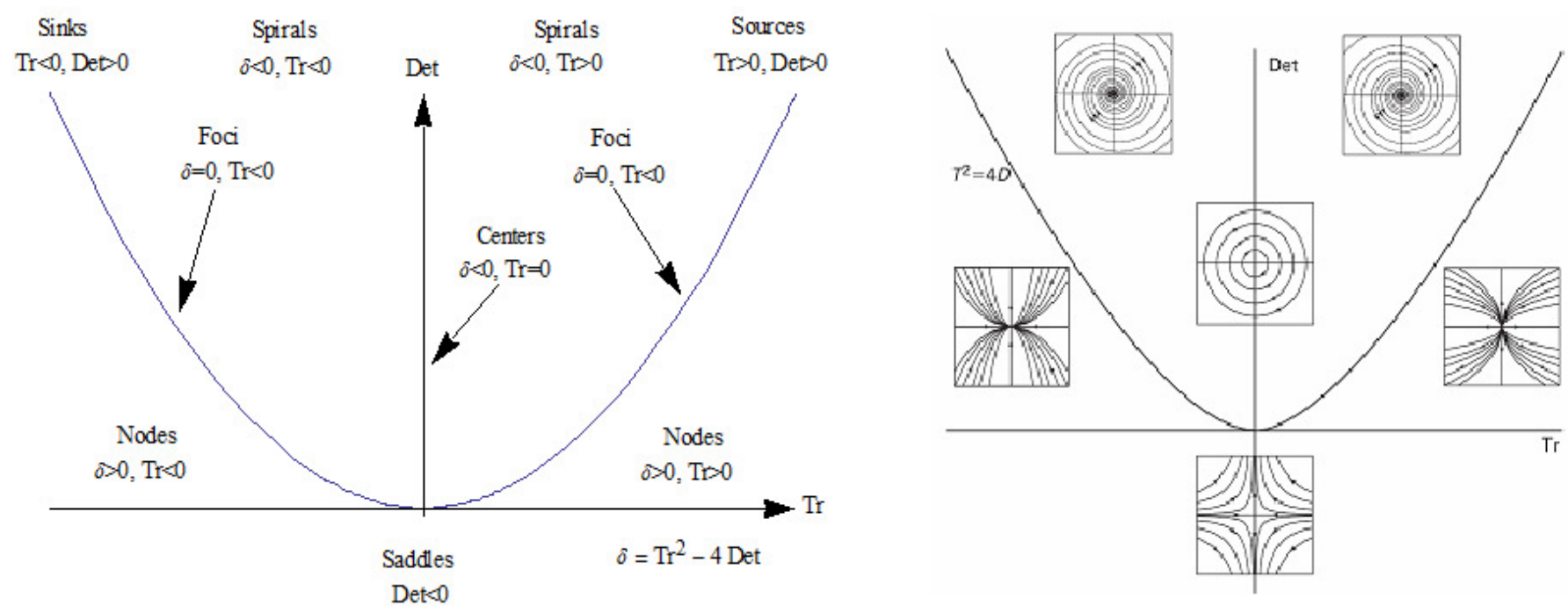

Figure 2. The trace-determinant plane.

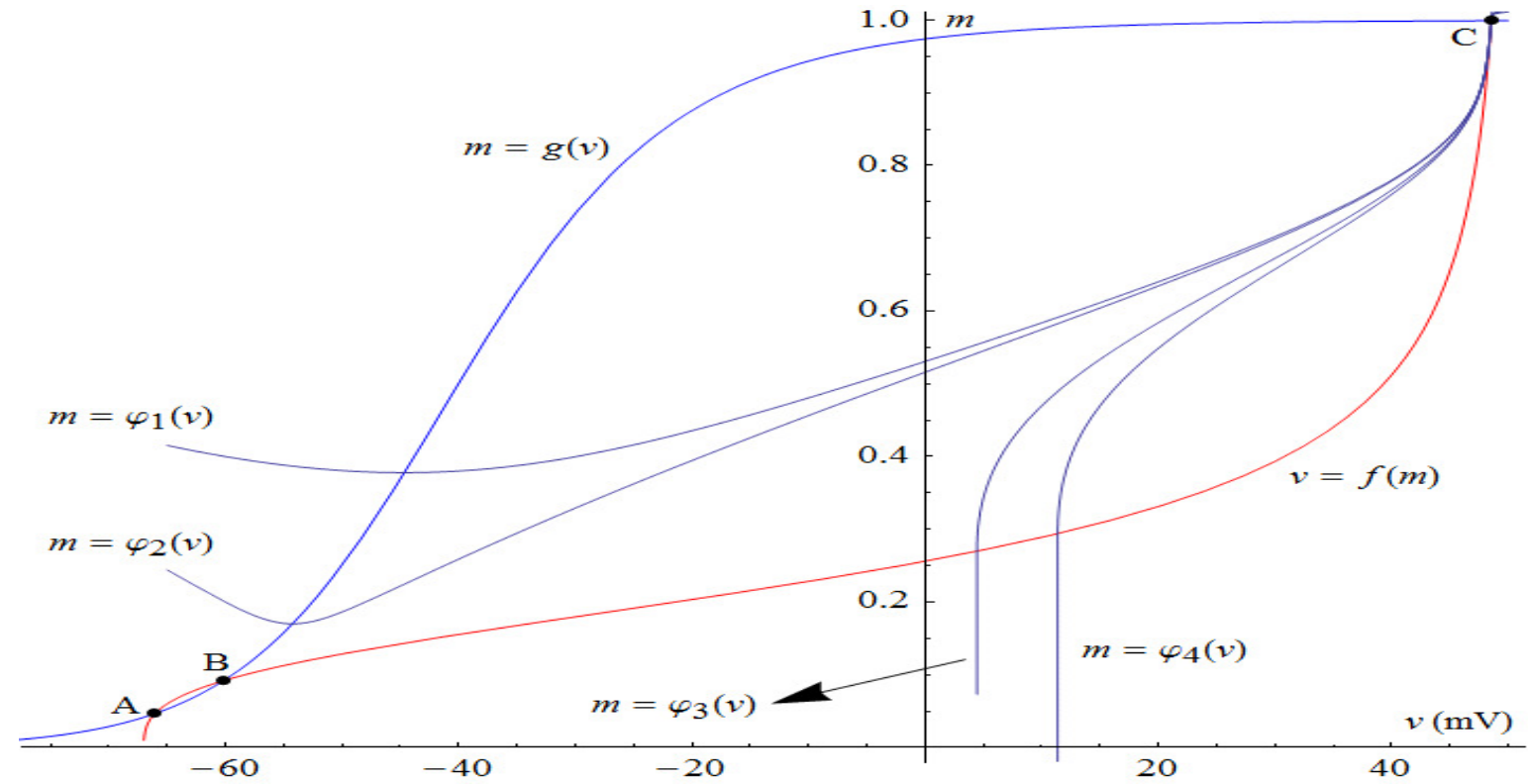

Figure 3. The Hodgkin-Huxley phase-plane, showing the nullclines $m=g(v)$ and $v=f(m)$ with $n 0=0.32$ and h0 = 0.45 ), four sample trajectories and three equilibrium point $A, B$ (sink nodes) and $C$ (saddle point).

$$
\left\{\begin{array}{l}
v=f(m)=\frac{E_{L} g_{L}+E_{K} n_{0}{ }^{4} \bar{g}_{K}+m^{3} E_{N a} h_{0} \bar{g}_{N a}}{g_{L}+n_{0}{ }^{4} \bar{g}_{K}+m^{3} h_{0} \bar{g}_{N a}} . \\
m=g(v)=\frac{e^{\frac{137}{18}+\frac{7 v}{45}}(40+v)}{40 e^{4+\frac{v}{10}}+40 e^{\frac{137}{18}+\frac{7 v}{45}}-40+e^{\frac{137}{18}+\frac{7 v}{45}} v} .
\end{array}\right.
$$

Figure 3 shows these curves for the values given by (16). These curves intersect each other at the equilibrium points:
A(-66.0474, 0.0467), B(-60.165, 0.0919), C(48.547, 0.9992)

\section{Phase-plane analysis}

Table 1 shows the classification of the three equilibrium points (22). We took into account (12), (13), (16), (17), (18) and Figure 2. The phase portrait near these equilibrium points are shown in Figures 4,5 and 6 . These portraits were obtained with the aid of Maple, version 15. The phase-planes in Figures 3 to 6 showed that there are three intersections that are steady states, two stable 
Table 1. Equilibrium points classification.

\begin{tabular}{lcccl}
\hline Point & Tr & Det & $\delta$ & Classification \\
\hline A(-66.0474, 0.0467) & -5.13 & 2.02 & 18.25 & Sink Node \\
B(-60.165, 0.0919) & -4.09 & -2.725 & 27.6 & Saddle \\
C(48.547, 0.9992) & -63.41 & 483.33 & 2087.23 & Sink Node \\
\hline
\end{tabular}

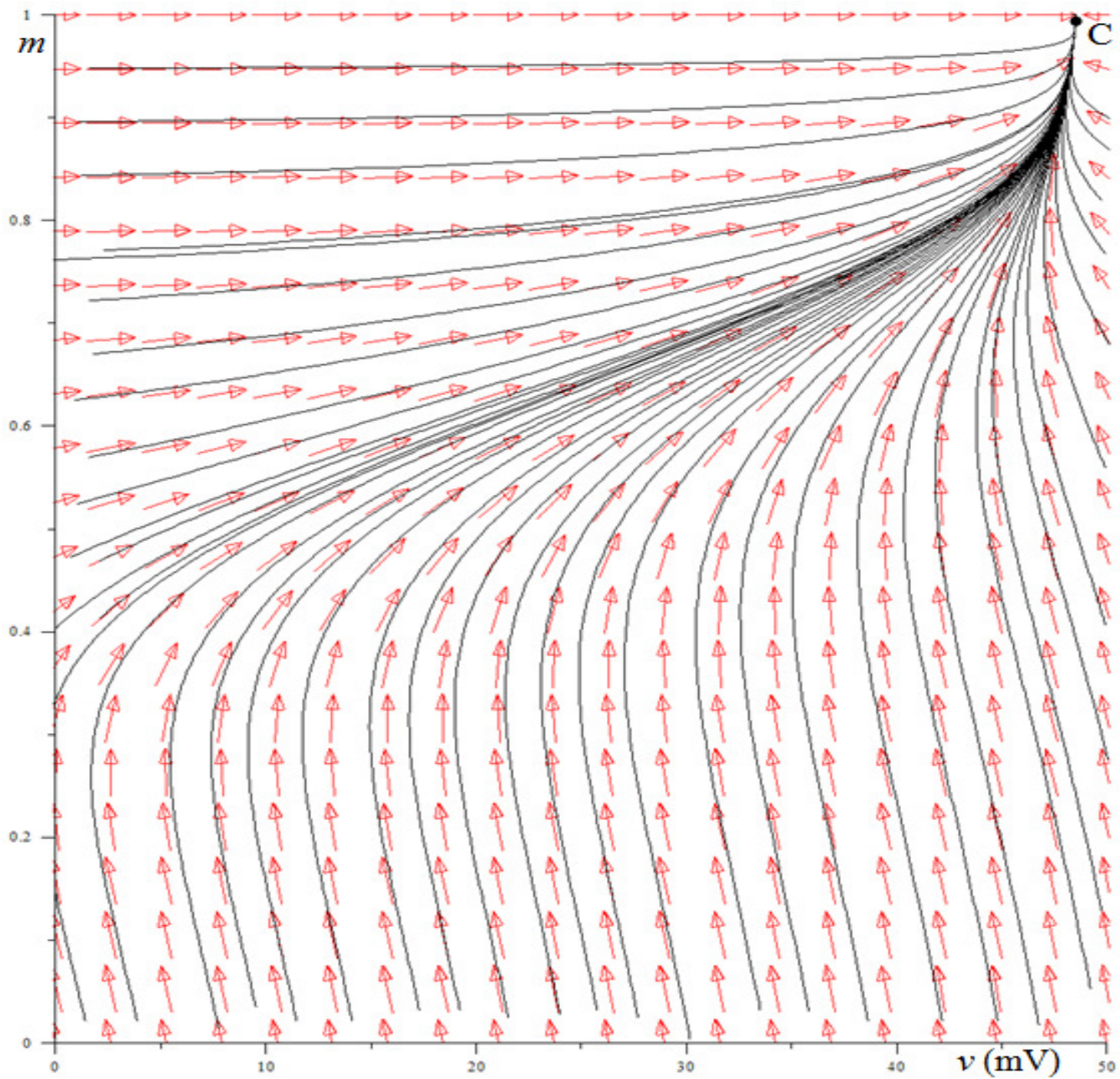

Figure 4. Phase portrait near the steady state C.

nodes $\mathrm{A}$ and $\mathrm{C}$ and one saddle point $\mathrm{B}$.

\section{RESULTS AND DICUSSION}

Point A (Figures 3, 5 and 6) corresponds to the cellular membrane resting potential and small deviations of this point do not lead to the membrane depolarization, but rather to a gradual return to the resting potential. The trajectories near this point but below the saddle point $B$ converge quickly to sink node $A$. If an impulse is larger than the threshold potential in the saddle point $B$, like it is shown with the sample trajectories in Figures 3, 5 and 6, the fast sodium channels start their opening and the 


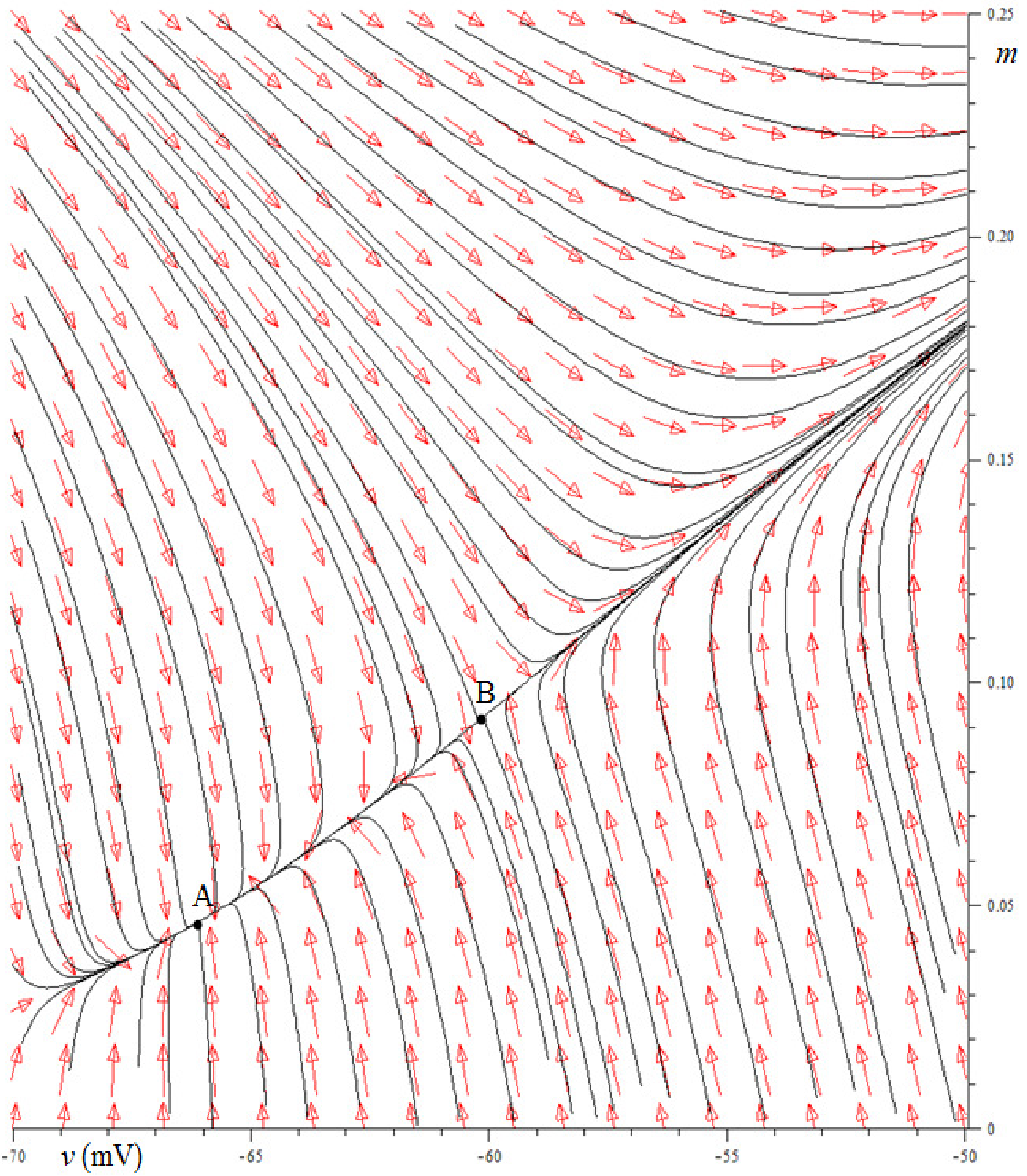

Figure 5. Phase portrait near the steady states $A$ and $B$.

membrane depolarization process is activated (influx of $\mathrm{Na}$ ions inside the membrane) due to their concentration gradient and the quickly opening of $\mathrm{Na}$ voltage-gated channels will lead the membrane potential to the 


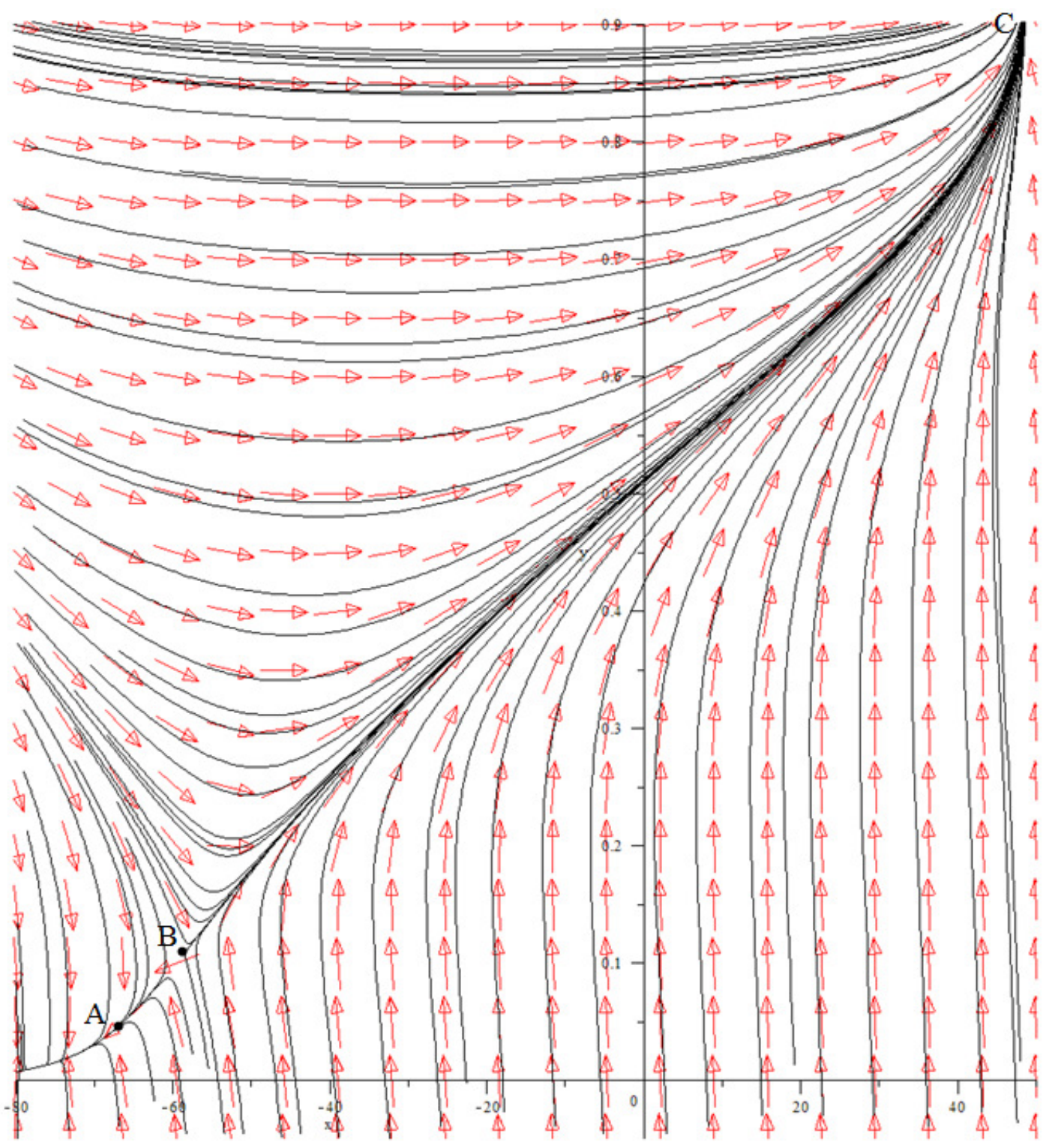

Figure 6. Global Phase Portrait (points A, B and C).

depolarization state as illustrated with the attracting point at sink point $\mathrm{C}$ (Figures 3, 4 and 6).

\section{REFERENCES}

Connor JA, Stevens CF (1971). Inward and delayed outward membrane currents in isolated neural somata under voltage clamp. J. Physiol. 213:1-19.

Doi S, Junko I, Pan Z, Tsumoto K (2010). Computational Electrophysilogy. Dynamical Systems and Bifurcations. Springer, Tokio.

Ebihara L, Johnson EA (1980). Fast sodium current in cardiac muscle. A quantitative description. Biophys. J. 32:779-790.

Edelstein-Keshet L (1988). Mathematical Models in Biology. Mc GrawHill. Unit States of America.

FitzHugh R (1969). Mathematical models of excitation and propagation in nerve, Biological Engineering, edited by H. P. Schwan, McGraw-
Hill, New York pp. 1-85.

Hille B (2001). Ionic channels of excitable membranes. Sinauer Associates, Sunderland, MA, $3^{\text {rd }}$ edition.

Hodgkin AL, Huxley AF (1952b). Current carried by sodium and potassium ions through the membrane of the giant axon of loligo. J. Physiol. 116:449-472.

Hodgkin AL, Huxley AF (1952c). The components of membrane conductance in the giant axon of Loligo. J. Physiol. 116:473-496.

Hodgkin AL, Huxley AF (1952d). The dual effect of membrane potential on sodium conductance in the giant axon of loligo. J. Physiol. 116:497-506.

Hodgkin AL, Huxley AF (1952e). A quantitative description of membrane current and its applications to conduction and excitation in nerve. J. Physiol. 117:500-544.

Hodgkin AL, Huxley AF, Katz B (A, 1952). Measurement of currentvoltage relations in the membrane of the giant axon of loligo. $\mathrm{J}$. Physiol. 116:424-448.

Keener J, Sneyd J (1998). Mathematical Physiology, Springer-Verlag, New York Inc. 
Luo CH, Rudy Y (1994). A dynamic model of the cardiac ventricular action potential. I. Simulations of ionic currents and concentration changes. Circ. Res. 74:1071-1096.

Nagumo J, Arimoto S, Yoshizawa S (1962). An active pulse transmission line simulating nerve axon. Proc. IRE. 50:2061-2070.

Schierwagen A (1991). Traveling wave solutions of a simple nerve conduction equation for inhomogeneous axons, Nonlinear Waves in Excitable Media, edited by A. V. Holden, M. Markus, and H. Othmer, Manchester University Press, Manchester pp. 107-114.
Scott A (2002). Neuroscience: A Mathematical Primer, Springer-Verlag, New York.

Waxman SG, Kocsis JD, Stys PK eds (1995). The Axon. Structure, Function and Pathophysiology. Oxford University Press, Oxford. 


\section{APPENDIX}

\section{Mathematica 7 code}

Clear $[\mathrm{v}, \mathrm{m}]$;

$\alpha_{m}\left[v_{-}\right]:=\frac{\frac{1}{10}(v+40)}{1-\operatorname{Exp}\left[-\frac{1}{10}(v+40)\right]} ;$

$\beta_{m}\left[v_{-}\right]:=4 \operatorname{Exp}\left[-\frac{v+65}{18}\right]$;

$\mathrm{C}_{\mathrm{m}}=1 ; \overline{\mathrm{g}}_{\mathrm{K}}=36 ; \mathrm{g}_{\mathrm{I}}=0.3 ; \overline{\mathrm{g}}_{\mathrm{Na}}=120 ; \mathrm{E}_{\mathrm{K}}=-77 ; \mathrm{E}_{\mathrm{I}}=-54.4 ; \mathrm{E}_{\mathrm{Na}}=50 ; \mathrm{n}_{0}=0.32 ;$

$\mathrm{h}_{0}=0.45 ; \mathrm{T} 1=0 ; \mathrm{T} 2=20 ; \mathrm{v}_{0}=-66 ; \mathrm{m}_{0}=0.01 ;$

Clear $[\mathrm{v}, \mathrm{m}]$;

sys $=\left\{v^{\prime}[t]=-\frac{1}{C_{m}}\left(\bar{g}_{N a} h_{0} m[t]^{3}\left(v[t]-E_{N a}\right)+\bar{g}_{K} n_{0}{ }^{4}\left(v[t]-E_{K}\right)+g_{I}\left(v[t]-E_{L}\right)\right)\right.$,

$\left.\mathrm{m}^{\prime}[\mathrm{t}]==\alpha_{\mathrm{m}}[\mathrm{v}[\mathrm{t}]](1-\mathrm{m}[\mathrm{t}])-\beta_{\mathrm{m}}[\mathrm{v}[\mathrm{t}]] * \mathrm{~m}[\mathrm{t}], \mathrm{v}[\mathrm{T} 1]==\mathrm{v}_{0}, \mathrm{~m}[\mathrm{~T} 1]==\mathrm{m}_{0}\right\} ;$

sol = NDSolve $[$ sys $,\{\mathrm{v}[\mathrm{t}], \mathrm{m}[\mathrm{t}]\},\{\mathrm{t}, \mathrm{T} 1, \mathrm{~T} 2\}]$;

$\mathrm{v}=\operatorname{sol}[[1,1,2]]$;

$\mathrm{m}=\operatorname{sol}[[1,2,2]]$; 